\title{
A Proposed Technique for Analyzing Experiments Of Type $2^{p} 3^{m}$
}

\author{
Abbas F. M. Alkarkhi (Corresponding author) \\ Environmental Technology Division \\ School of Industrial Technology \\ Universiti Sains Malaysia \\ 11800 Penang, Malaysia \\ Tel.: 60-4-653-2107Ｅ-mail: alkarkhi@yahoo.com \\ H. C. Low \\ School of Mathematical Sciences \\ Universiti Sains Malaysia \\ 11800 Penang, Malaysia \\ Tel: 60-4-653-3641Ｅ-mail: hclow@cs.usm.my
}

\begin{abstract}
This paper deals with the experiments of type $2^{p} 3^{m}$. Fitting a response surface model to this type of experiments was studied using a new technique. This technique depends on partitioning the whole experiment into two experiments and analyzes each experiment separately. In addition to the proposed technique, the least squares method was studied and a comparison is made between the proposed technique and the least squares method. The results showed that the proposed technique and least squares method gave the same results. This indicates that partitioning the whole experiment does not affect the results of fitting the response surface model. This paper proposes an easy technique for analyzing this type of experiments. The results showed that the new technique is easier to use than the least squares method. Simple calculations and substitutions in fixed formulae are used to get the results directly. On the contrary, the least squares method requires complicated calculations to get the results.
\end{abstract}

Keywords: Response surface, Contrast, Least squares method

\section{Introduction}

Experiments are performed by investigators in virtually all fields of inquiry, usually to discover something about a particular process or system. Factorial designs are widely used in experiments involving several factors where it is necessary to study the joint effects of the factors on the response.

Experiments of type $2^{p} 3^{m}$ have been used in many disciplines, especially in industry where there are several factors; some of the factors are at two levels, and others at three levels. This type of experiment consists of $p+m$ factors, where $p$ factors have two levels, and $m$ factor have three levels (Montgomery, 2002). Margolin (1967) proposed a new procedure for analyzing this type of experiments $2^{p} 3^{m}$, how to calculate the effects, sum of squares and fitting a response surface model. The technique is based on the two procedures presented by Yates (1964) for two and three levels. In this paper, the factors are denoted by capital letters $A, B, \ldots$, and the levels are denoted by -1 if the factor is at the low level and 1 if the factor is at the high level in the case of two levels, and for factors with three levels -1 if the factor is at the low level, 0 if the factor is at the intermediate level, and 1 if the factor is at the high level. The objective of this study is to provide an alternative technique for fitting a response surface model to the experiments of type $2^{p} 3^{m}$.

\section{Fitting a response surface model to an experiment of type $2^{p} 3^{m}$}

Consider the following response surface model for an experiment of type $2^{p} 3^{m}$

$Y=\beta_{0}+\sum_{j=1}^{p} \beta_{j} X_{j}+\sum_{l=1}^{m} \gamma_{l} Z_{l}+\sum_{l=1}^{m} \gamma_{l l} Z_{l l}^{2}+\sum_{j<i} X_{i} X_{j}+\sum_{l<q} \gamma_{l q} Z_{l} Z_{q}+\sum_{j=1}^{p} \sum_{l=1}^{m} \delta_{j l} X_{j} Z_{l}$

where 
$X_{j}$ represents factors with two levels, and $Z_{l}$ represents factors with three levels.

The technique of least squares is used to estimate the parameters of the model. In this technique, we minimize the sum of the squared differences of the actual $Y$ values and the values of $Y$ predicted by the regression equation. The computation can be complicated and difficult if the number of factors included in the model is large.

\subsection{The proposed technique}

The proposed technique depends on partitioning the whole experiment $2^{p} 3^{m}$ into two experiments. To fit a response surface model to this type of experiment, the following steps are used:

(1) The first step is to partition the experiment $\left(2^{p} 3^{m}\right)$ into two experiments according to the number of levels, one experiment with factors at three levels $3^{m}$ and the other with factors at two levels $2^{p}$.

(2) Analyze each experiment separately:

For two levels, the procedure given by Abbas et al., (2000) is used as follows:

$b_{j}=\frac{\text { Contrast for } A_{j}}{4 n}$

$b_{j i}=\frac{\text { Contrast for } A_{j} A_{i}}{4 n}$,

$j<i$

And for three levels, the procedure given by Abbas et al., (2001) is used as follows:

$$
\begin{array}{ll}
\gamma_{l}=\frac{\text { Linear contrast for } A_{l}}{2 n}, & \gamma_{l l}=\frac{\text { Quadratic contrast for } A_{l}}{2 n} \\
\gamma_{l q}=\frac{\text { Linear contrast for } A_{l} A_{q}}{4 n}, & l<q
\end{array}
$$

(3) For this step, $C_{1}^{p} C_{1}^{m}$ experiments of the form $2^{1} 3^{1}$ are constructed to find the interaction between factors that have three levels and factors that have two levels.

To fit a response surface model to the experimental results, the formulae in step 2 are used, and for the joint coefficients, the following formula shall be used:

$$
\delta_{j l}=\frac{C L}{2\left(n_{1}+n_{2}\right)}=\frac{C L}{4 n}, \quad\left(n_{1}=n_{2}=n\right), \quad j=1,2, \ldots, p, \quad l=1,2, \ldots, m
$$

where

$\delta_{i l} \quad$ represents the regression coefficient for factor $l$ which has three levels and factor $q$ which has two levels,

$n_{1} \quad$ represents the number of replicates at the low level for the factor at two levels,

$n_{2} \quad$ represents the number of replicates at the high level for the factor at two levels, and

(4) represents the distance between the high and low levels of the three level factor.

Also,

$$
\begin{aligned}
& C L=d_{+1}-d_{-1}, \text { where } \\
& d_{+1} \quad \begin{array}{l}
\text { represents the joint contrast between the factor at three levels and the high } \\
\text { level for the factor at two levels, and }
\end{array} \\
& \begin{array}{l}
\text { represents the joint contrast between the factor at three levels and the low } \\
\text { level for the factor at two levels. }
\end{array}
\end{aligned}
$$

Furthermore, the joint coefficient can be found by using the following formula:

$$
\delta_{j l}=\frac{C L}{n \sum C_{j l}^{2}}=\frac{C L}{4 n}, \quad l=1,2, \ldots, p, \quad l=1,2, \ldots, m
$$

where

$$
\sum C_{j l}^{2} \text { represents the sum of squares of the joint contrast coefficients, }
$$

and $C L$ represents the linear contrast, and 
n represents the number of replicates

(5) To find the sum of squares, the following formula is used:

$$
\text { S.S. }=\frac{C L^{2}}{2\left(n_{1}+n_{2}\right)}=\frac{C L^{2}}{4 n}, \quad\left(n_{1}=n_{2}=n\right)
$$

\subsection{Prooffor the $2^{p} 3^{m}$ experiments}

Consider the following model:

$$
Y=\beta_{0}+\sum_{j=1}^{p} \beta_{j} X_{j}+\sum_{l=1}^{m} \gamma_{l} Z_{l}+\sum_{l=1}^{m} \gamma_{l l} Z_{l l}^{2}+\sum_{j<i} X_{i} X_{j}+\sum_{l<q} \gamma_{l q} Z_{l} Z_{q}+\sum_{j=1}^{p} \sum_{l=1}^{m} \delta_{j l} X_{j} Z_{l}
$$

subjected to the following constraints:

2.2.1 For the factors at two levels:

$$
\begin{aligned}
\sum_{i=1}^{k} X_{i}=0, \quad \sum_{i<j}^{k} X_{i} X_{j}=0, \sum_{i<j}^{k} X_{i}^{2} X_{j}=0, \quad \sum_{i<j}^{k} X_{i} X_{j}^{2}=0 \\
\sum_{i=1}^{k} X_{i}^{2}=k, \sum_{i<j}^{k}\left(X_{i} X_{j}\right)^{2}=k
\end{aligned}
$$

These constraints come from factor levels related to the factors having two levels.

2.2.2 For factors at three levels

$$
\begin{array}{r}
\sum_{i=1}^{k} Z_{i}=0, \quad \sum_{i<j}^{k} Z_{i} Z_{j}=0, \quad \sum_{i<j}^{k} Z_{i} Z_{j}^{2}=0, \quad \sum_{i=1}^{k} Z_{i}^{4}=\sum_{i=1}^{k} Z_{i}^{2}=2 \times 3^{m-1}, \\
\sum_{i<j}^{k}\left(Z_{i} Z_{j}\right)^{2}=\sum_{i<j}^{k}\left(Z_{i} Z_{j}\right)^{2}=4 \times 3^{m-2},
\end{array}
$$

These constraints come from factor levels related to the factors having three levels.

2.2.3 Constraints related with mixed factors, some factors have two levels and others have three levels

$$
\begin{aligned}
& \sum_{i=1}^{k}\left(Z_{i} X_{i}\right)^{2}=4 \times\left(3^{m-1} \times 2^{p-1}\right), \sum_{i=1}^{k} X_{i} Z_{i}=\sum_{i=1}^{k} X_{i}^{2} Z_{i}=\sum_{i=1}^{k} X_{i} Z_{i}^{2}=0, \\
& \sum_{i<j<w}^{k} X_{i} X_{j} Z_{w}=\sum_{i<j<w}^{k} Z_{i} Z_{j} X_{w}=0 .
\end{aligned}
$$

These constraints come from the mixed factors.

To illustrate the procedure, consider a $2^{2} 3^{2}$ experiment.

Suppose there are four factors: $Z_{1}, Z_{2}$ at three levels and $X_{1}, X_{2}$ at two levels (Table 1). The model for this experiment is:

$$
\begin{gathered}
Y=b_{0}+b_{1} X_{1}+b_{2} X_{2}+b_{12} X_{1} X_{2}+\gamma_{1} Z_{1}+\gamma_{2} Z_{2}+\gamma_{11} Z_{1}^{2}+\gamma_{22} Z_{2}^{2}+\gamma_{12} Z_{1} Z_{2}+\delta_{11} Z_{1} X_{1}+\delta_{12} Z_{1} X_{2} \\
+\delta_{21} Z_{2} X_{1}+\delta_{22} Z_{2} X_{2}
\end{gathered}
$$

For the linear and quadratic coefficients and the linear interaction for $3^{k}$, we obtain the same results that we obtained previously. The same results are also obtained for $2^{p}$.

Procedure: 
(1) First, partition this experiment into two experiments as follows:

a. Experiments where the factors have three levels.

b. Experiments where the factors have two levels.

(2) Analyze each experiment separately as mentioned in section 2.1.

The formula for the interaction coefficient between a factor at two levels and a factor at three levels is given below:

If equation ( 5 ) is multiplied by $Z_{1} X_{1}$, and sum over $\mathrm{i}$, then

$\sum Z X_{1} Y=\delta_{11} \sum\left(Z_{1} X_{1}\right)^{2}$

$\delta_{11}=\frac{\sum Z_{1} X_{1} Y}{\sum\left(Z_{1} X_{1}\right)^{2}}$

Similarly, for $\delta_{12}, \delta_{21}$ and $\delta_{22}$

In general, the formula is given by:

$\delta_{j l}=\frac{\sum Z_{l} X_{j} Y}{\sum\left(Z_{l} X_{j}\right)^{2}}$

Consider the formula for $\delta_{11}$ :

$\delta_{11}=\frac{\sum Z_{1} X_{1} Y}{\sum\left(Z_{1} X_{1}\right)^{2}}$

The denominator

$\sum\left(Z_{1} X_{1}\right)^{2}=4 \times\left(3^{m-1} \times 2^{p-1}\right)=4 \times\left(3^{2-1} \times 2^{2-1}\right)=4 \times 6=24$, This is equal to $4 \times 6$, where 6 represents the number of replicates at each joint level.

The numerator

$$
\begin{aligned}
& \sum_{i=1}^{36} Z_{1 i} X_{1 i} Y_{i}=(-1)(-1) Y_{1}+(0)(1) Y_{2}+(1)(1) Y_{3}+\ldots+(-1)(1) Y_{16}+(0)(1) Y_{17}+(1)(1) Y_{18}+ \\
&(-1)(1) Y_{19}+(0)(1) Y_{20}+(1)(1) Y_{21}+\ldots+(-1)(1) Y+(0)(1) Y+(1)(1) Y_{36} \\
&=\left(Y_{1}+Y_{4}+Y_{7}+Y_{10}+Y_{13}+Y_{16}+Y_{21}+Y_{24}+Y_{27}+Y_{30}+Y_{33}+Y_{36}\right)+ \\
&(0)\left(Y_{2}+Y_{5}+Y_{8}+Y_{11}+Y_{14}+Y_{17}+Y_{20}+Y_{23}+Y_{26}+Y_{29}+Y_{32}+Y_{35}\right)- \\
&\left(Y_{3}+Y_{6}+Y_{9}+Y_{12}+Y_{15}+Y_{18}+Y_{19}+Y_{22}+Y_{25}+Y_{28}+Y_{31}+Y_{34}\right)
\end{aligned}
$$

(3) In the third step, consider experiments where one factor has three levels and the other has two levels (Table 2), and then sum the observations in the cells, which have the same joint level, before doing anything. For instance,

from Table 2, it can be seen that

$$
\begin{gathered}
d_{-1}=(-1)(-1)\left(Y_{1}+Y_{10}+Y_{4}+Y_{13}+Y_{7}+Y_{16}\right)+(0)(-1)\left(Y_{2}+Y_{11}+Y_{5}+Y_{14}+Y_{8}+Y_{17}\right)+ \\
(1)(-1)\left(Y_{3}+Y_{12}+Y_{6}+Y_{15}+Y_{9}+Y_{18}\right)
\end{gathered}
$$

and

$$
\begin{aligned}
& d_{+1}=(-1)(1)\left(Y_{19}+Y_{28}+Y_{22}+Y_{31}+Y_{25}+Y_{34}\right)+(0)(1)\left(Y_{20}+Y_{29}+Y_{23}+Y_{32}+Y_{26}+Y_{35}\right)+ \\
& (1)(1)\left(Y_{21}+Y_{30}+Y_{24}+Y_{33}+Y_{27}+Y_{36}\right) \\
& \therefore C L=d_{+1}-d_{-1} \\
& =\left(Y_{1}+Y_{4}+Y_{7}+Y_{10}+Y_{13}+Y_{16}+Y_{21}+Y_{24}+Y_{27}+Y_{30}+Y_{33}+Y_{36}\right)+ \\
& (0)\left(Y_{2}+Y_{5}+Y_{8}+Y_{11}+Y_{14}+Y_{17}+Y_{20}+Y_{23}+Y_{26}+Y_{29}+Y_{32}+Y_{35}\right)-
\end{aligned}
$$




$$
\left(Y_{3}+Y_{6}+Y_{9}+Y_{12}+Y_{15}+Y_{18}+Y_{19}+Y_{22}+Y_{25}+Y_{28}+Y_{31}+Y_{34}\right)=\sum Z_{1} X_{1} Y
$$

This is equal to the numerator of equation (6).

The joint contrast can also be found by using the contrast directly, as follows:

$$
\begin{gathered}
C L=(-1)(-1)\left(Y_{1}+Y_{10}+Y_{4}+Y_{13}+Y_{7}+Y_{16}\right)+(-1)(1)\left(Y_{19}+Y_{28}+Y_{22}+Y_{31}+Y_{25}+Y_{34}\right)+ \\
(0)(-1)\left(Y_{2}+Y_{11}+Y_{5}+Y_{14}+Y_{8}+Y_{17}\right)+(0)(1)\left(Y_{20}+Y_{29}+Y_{23}+Y_{32}+Y_{26}+Y_{35}\right)+ \\
(1)(-1)\left(Y_{3}+Y_{12}+Y_{6}+Y_{15}+Y_{9}+Y_{18}\right)+(1)(1)\left(Y_{21}+Y_{30}+Y_{24}+Y_{33}+Y_{27}+Y_{36}\right)=\sum Z_{1} X_{1} Y
\end{gathered}
$$

Thus,

$$
\delta_{11}=\frac{\text { Linear contrast for } Z_{1} X_{1}}{4 \times 6}=\frac{\text { Linear contrast for } Z_{1} X_{1}}{4 \times n} \quad(\text { Here } \mathrm{n}=6)
$$

Note that 6 represent the number of replicates.

Similarly for $\delta_{12}, \delta_{21}$ and $\delta_{22}$.

In general, if we let $\mathrm{n}$ represent the number of replicates, the formula becomes:

$$
\delta_{j l}=\frac{\text { Linear contrast for } A_{j} A_{l}}{4 \times n}, \quad j=1,2, \ldots, p, \quad l=1,2, \ldots, m
$$

\section{Application}

Suppose the situation where there are three factors with two of them having two levels and the other factor having three levels (Margolin, 1967). Table 3 presents three factors: $X_{1}$ and $X_{2}$ with two levels, and $Z_{1}$ with three levels.

The least squares method and the new procedure will be used to fit a response surface model to the experiment:

\subsection{The least squares method}

SPSS version 11 was used to obtain the results as shown below:

$$
\hat{Y}=20-9 X_{1}+10 X_{2}+4 X_{1} X_{2}+8 X_{3}+15 X_{3}^{2}-5 X_{3} X_{1}+7 X_{3} X_{2}
$$

\subsection{The proposed technique}

Use the procedure explained in Section 2.1 for analyzing this experiment and then compares the results with the least squares method. The following gives the procedure step by step:

Step 1. Analysis of the $3^{1}$ experiment (Table 3.a):

Linear contrast,

$$
C L=(-1) 108+0(80)+(1) 172=64
$$

To find the coefficient of regression, $\gamma_{1}$, the following formula was used

$\gamma_{1}=\frac{C L}{2 n}=\frac{64}{2(4)}=8$

Quadratic contrast,

$$
C Q=(1) 108+(-2) 80+(1) 172=120
$$

To find the coefficient of regression, $\gamma_{11}$, The following formula was used

$\gamma_{1}=\frac{C Q}{2 n}=\frac{120}{2(4)}=15$

Step 2. Analysis of the $2^{2}$ experiment (Table 3.b):

Let $X_{1}=A, X_{2}=B$, then

$A=(a-1)(b+1)=105+21-99-135=-108$

$b_{1}=\frac{\text { Contrast for } A}{4 n}=\frac{-108}{4 \times 3}=-9$ 


$$
\begin{aligned}
& B=(a+1)(b-1)=105+135-21-99=120 \\
& \therefore b_{2}=\frac{\text { Contrast for } B}{4 n}=\frac{120}{4 \times 3}=10 \\
& A B=(a-1)(b-1)=105+99-135-21=48 \\
& b_{12}=\frac{\text { contrast for } A B}{4 n}=\frac{84}{4 \times 3}=4
\end{aligned}
$$

Step 3. Analysis of the $3^{1} 2^{1}$ experiments (Table 3.c and Table 3.d):

The contrast between the factor at three levels and the lower level for the factor at two levels was found as follows:

$$
d_{-1}=(-1) 64+(0) 54+(1) 116=52
$$

Next, the contrast between the factor at three levels and the higher level for the factor at two levels is given below:

$$
d_{+1}=(-1)(44)+(0) 26+(1) 56=12
$$

The linear contrast is equal to:

$$
\begin{aligned}
& C L=d_{+1}-d_{-1}=12-52=-40 \\
& \delta_{11}=\frac{C L}{2\left(n_{1}+n_{2}\right)}=\frac{C L}{4 n}=\frac{-40}{4(2)}=-5 \quad, \quad 4 n=4 \times\left(3^{m-1} \times 2^{p-1}\right)=4 \times\left(3^{1-1} \times 2^{2-1}\right)=4 \times 2=8
\end{aligned}
$$

Or by using the alternative formula:

$$
\begin{aligned}
\delta_{31} & =\frac{C L}{n \sum C_{i j}^{2}}=\frac{C L}{4 n} \\
C L=(0)(0)(64)+(0)(1)(44)+\ldots+(2)(1) 56=-40 & \\
\delta_{31}=\frac{-40}{4(2)} & =-5
\end{aligned}
$$

For $\delta_{21}$,

$$
\begin{array}{cc}
d_{-1}=-42+0+46=4, & d_{+1}=-66+0+126=60 \\
C L=60-4=56 &
\end{array}
$$$$
\delta_{21}=\frac{C L}{4 n}=\frac{56}{4(2)}=7
$$

Or by using the formula:

$$
\begin{aligned}
& \delta_{21}=\frac{C L}{n \sum C_{i j}^{2}}=\frac{C L}{4 n} \\
& C L=(0)(0)(42)+\ldots+(2)(1)(126)=56 \\
& \delta_{21}=\frac{56}{4(2)}=7 \\
& b_{0}=\bar{Y}-b_{33} \bar{X}_{3}^{2}=\frac{360}{12}-15 \frac{8}{12}=30-10=20
\end{aligned}
$$

Comparing the results obtained by least squares and the new procedure (Table 4), it can be seen that the results obtained by the proposed technique and the least squares method are the same.

\section{Conclusion}

It can be concluded that the least squares method and the proposed technique gave the same values for the coefficients of the model. It may also be concluded that partitioning an experiment of type $2^{p} 3^{m}$ into two-experiment does not affect the results. The new technique is easier to use than the least squares method, as the results can be obtained directly from simple calculations and substitutions in fixed formulae. On the contrary, the least squares method requires complicated calculations to get the results. 


\section{References}

Abbas F. M. A., Low, H. C. and Quah, S. H., (2000). A New Method For Analyzing Experiment of Type $2^{\mathrm{p} " .}$ National Conference on Management Science/ Operations Research, November (2000). Malaysia. pp 123-130.

Abbas F. M. A., Low, H. C. and Quah, S. H., (2001). A New Procedure for Fitting a Response Surface Model To Experiments of Type $3^{\mathrm{m}}$. International Conference on Recent Developments in Statistics and its Applications, Malaysia. pp 1-8.

Draper N.R \& smith, H., (1998). Applied regression Analysis $3^{\text {rd }}$ edition. Wiley.

Margolin, B. H. (1967). "Systematic Methods of Analysis 2 $3 \mathrm{k}$ Factorial Experiments with Applications." Technometrics, Vol. 9, pp. 245-260.

Montgomery, D. C. (2002). Design and Analysis of Experiments. $5^{\text {th }}$ edition, New York: John Wiley \& Sons, Inc.

Yates, F. (1964).The design and Analysis of Factorial Experiments. $5^{\text {th }}$ edition, Harpenden, England: Publisher (Rothmasted Experimental Station). Technical Communication, No. 35 of the Commonwealth Bureau of Soils.

Table 1. A design with four factors where $Z_{1}, Z_{2}$ have three levels each, and $X_{1}, X_{2}$ have three levels each

\begin{tabular}{|c|c|c|c|c|}
\hline \multirow{4}{*}{$\mathrm{Z}_{1}$} & \multirow{4}{*}{$\mathrm{Z}_{2}$} & \multirow{4}{*}{$\begin{array}{l}\mathrm{X}_{1} \\
\mathrm{X}_{2}\end{array}$} & -1 & -1 \\
\hline & & & 1 & 1 \\
\hline & & & -1 & 1 \\
\hline & & & -1 & 1 \\
\hline-1 & -1 & & $\mathrm{Y}_{1}$ & $\mathrm{Y}_{10}$ \\
\hline 0 & -1 & & $\mathrm{Y}_{19}$ & $\mathrm{Y}_{28}$ \\
\hline 1 & -1 & & $\mathrm{Y}_{2}$ & $\mathrm{Y}_{11}$ \\
\hline-1 & 0 & & $\mathrm{Y}_{20}$ & $\mathrm{Y}_{29}$ \\
\hline & & & $\mathrm{Y}_{3}$ & $\mathrm{Y}_{12}$ \\
\hline 0 & 0 & & $\mathrm{Y}_{21}$ & $\mathrm{Y}_{30}$ \\
\hline 1 & 0 & & $\mathrm{Y}_{4}$ & $Y_{13}$ \\
\hline-1 & 1 & & $\mathrm{Y}_{22}$ & $Y_{31}$ \\
\hline 0 & 1 & & $\mathrm{Y}_{5}$ & $\mathrm{Y}_{14}$ \\
\hline 1 & 1 & & $\mathrm{Y}_{23}$ & $Y_{32}$ \\
\hline & & & $Y_{6}$ & $\mathrm{Y}_{15}$ \\
\hline & & & $\mathrm{Y}_{24}$ & $Y_{33}$ \\
\hline & & & $\mathrm{Y}_{7}$ & $Y_{16}$ \\
\hline & & & $\mathrm{Y}_{25}$ & $\mathrm{Y}_{34}$ \\
\hline & & & $Y_{8}$ & $Y_{17}$ \\
\hline & & & $\mathrm{Y}_{26}$ & $\mathrm{Y}_{35}$ \\
\hline & & & $\mathrm{Y}_{9}$ & $\mathrm{Y}_{18}$ \\
\hline & & & $Y_{27}$ & $\mathrm{Y}_{36}$ \\
\hline
\end{tabular}

Table 2. The results for factors $Z_{1}$ at three levels and $X_{1}$ at two levels

\begin{tabular}{|c|c|c|}
\hline $\begin{array}{ll} & \mathrm{X}_{1} \\
\mathrm{Z}_{1} & \end{array}$ & -1 & 1 \\
\hline-1 & $\mathrm{Y}_{1}+\mathrm{Y}_{10}+\mathrm{Y}_{4}+\mathrm{Y}_{13}+\mathrm{Y}_{7}+\mathrm{Y}_{16}$ & $\mathrm{Y}_{19}+\mathrm{Y}_{28}+\mathrm{Y}_{22}+\mathrm{Y}_{31}+\mathrm{Y}_{25}+\mathrm{Y}_{34}$ \\
\hline 0 & $\mathrm{Y}_{2}+\mathrm{Y}_{11}+\mathrm{Y}_{5}+\mathrm{Y}_{14}+\mathrm{Y}_{8}+\mathrm{Y}_{17}$ & $\mathrm{Y}_{20}+\mathrm{Y}_{29}+\mathrm{Y}_{23}+\mathrm{Y}_{32}+\mathrm{Y}_{26}+\mathrm{Y}_{35}$ \\
\hline 1 & $\mathrm{Y}_{3}+\mathrm{Y}_{12}+\mathrm{Y}_{6}+\mathrm{Y}_{15}+\mathrm{Y}_{9}+\mathrm{Y}_{18}$ & $\mathrm{Y}_{21}+\mathrm{Y}_{30}+\mathrm{Y}_{24}+\mathrm{Y}_{33}+\mathrm{Y}_{27}+\mathrm{Y}_{36}$ \\
\hline
\end{tabular}


Table 3 . The design matrix and results for the $2^{2} 3^{1}$ experiment

\begin{tabular}{cccccc}
\hline & $\mathrm{X}_{1}$ & -1 & -1 & 1 & 1 \\
$\mathrm{Z}_{1}$ & $\mathrm{X}_{2}$ & -1 & 1 & -1 & 1 \\
\hline-1 & & 28 & 36 & 14 & 30 \\
0 & & 27 & 27 & 5 & 21 \\
1 & & 44 & 72 & 2 & 54 \\
\hline
\end{tabular}

Table 3 a. The results for factor $\mathrm{X}_{3}$ at three levels

\begin{tabular}{lccc}
\hline $\mathrm{Z}_{1}$ Levels & Response & $\mathrm{L}$ & $\mathrm{Q}$ \\
\hline-1 & 108 & -1 & 1 \\
0 & 80 & 0 & -2 \\
1 & 172 & 1 & 1 \\
\hline
\end{tabular}

Table $3 \mathrm{~b}$. The results for factors $\mathrm{X}_{1}$ and $\mathrm{X}_{2}$, each at two levels

\begin{tabular}{|c|c|c|}
\hline $\begin{array}{ll} & \mathrm{X}_{2} \\
\mathrm{X}_{1} & \end{array}$ & -1 & 1 \\
\hline-1 & 99 & 135 \\
\hline 1 & 21 & 105 \\
\hline
\end{tabular}

Table $3 \mathrm{c}$. The results for factor $\mathrm{X}_{1}$ at two levels and factor $\mathrm{Z}_{1}$ at three levels

\begin{tabular}{|c|c|c|c|}
\hline $\begin{array}{ll} & X_{1} \\
Z_{1} & \end{array}$ & -1 & 1 & $\mathrm{~L}$ \\
\hline-1 & 64 & 44 & -1 \\
\hline 0 & 54 & 26 & 0 \\
\hline 1 & 116 & 56 & 1 \\
\hline
\end{tabular}

Table $3 \mathrm{~d}$. The results for factors $\mathrm{X}_{2}$ at two levels and factor $Z_{1}$ at three levels

\begin{tabular}{|c|c|c|c|}
\hline $\begin{array}{ll}\mathrm{X}_{2} & \\
\mathrm{Z}_{1} & \end{array}$ & -1 & 1 & $\mathrm{~L}$ \\
\hline-1 & 42 & 66 & -1 \\
\hline 0 & 32 & 48 & 0 \\
\hline 1 & 42 & 126 & 1 \\
\hline
\end{tabular}

Table 4. Results for the two methods

\begin{tabular}{|c|c|c|}
\hline Coefficient & Least squares & Proposed Procedure \\
\hline$b_{0}$ & 20 & 20 \\
\hline$b_{1}$ & -9 & -9 \\
\hline$b_{2}$ & 10 & 10 \\
\hline$b_{12}$ & 8 & 8 \\
\hline$\gamma_{1}$ & 15 & 15 \\
\hline$\gamma_{11}$ & -5 & -5 \\
\hline$\delta_{11}$ & 7 & 7 \\
\hline$\delta_{21}$ & 4 & 4 \\
\hline
\end{tabular}

